Available online at GSC Online Press Directory

GSC Biological and Pharmaceutical Sciences

e-ISSN: 2581-3250, CODEN (USA): GBPSC2

Journal homepage: https://www.gsconlinepress.com/journals/gscbps

(REVIEW ARTICLE)

\title{
Nanoparticles loaded in consumable polymeric oral films: A smart dosage form design for oral administration
}

\author{
Suhani Sinha* \\ Department of Pharmacy, School of Medical and Allied Sciences, G D Goenka University, Gurugram-122103, India.
}

Publication history: Received on 24 May 2020; revised on 04 July 2020; accepted on 06 July 2020

Article DOI: https://doi.org/10.30574/gscbps.2020.12.1.0153

\begin{abstract}
This review article provides an overview of nanoparticles in polymeric oral thin films as smart, non-parenteral and noninvasive route of drug administration to achieve patient compliance for varied therapeutic applications. The increasing research trend towards developing biocompatible and biodegradable nanoparticles as sustained release mucoadhesive patches with potential to improve pharmacokinetics and bioavailability significantly has given impetus to drug repurposing of existing molecules to new boundaries. Most widely used polymers to prepare nanoparticles include alginate and chitosan as they are biodegradable, biocompatible, demonstrated low toxicity in in vitro and in vivo models and has already been approved for dietary use and wound dressing applications. Chitosan particularly has been used as a carrier for polymeric nanoparticles for drug delivery through various routes of administration. Nanoparticles (NP) prepared using chitosan and chitosan derivatives typically possess a positive surface charge and has mucoadhesive properties such that they can adhere to mucus membranes and release the drug in a sustained release manner. They have various applications for the treatment of cancer, gastrointestinal diseases, pulmonary diseases, drug delivery to the brain and ocular infections through non-parenteral route of drug delivery.
\end{abstract}

Keywords: Oral film; Nanoparticles; Nanopharmaceutical; Nanodrugs; Chitosan; FDA approved; Bioavailability; Permeability; Dissolution.

\section{Introduction}

Majority of leads identified through the drug discovery process as potential new candidates capable of binding to specific target receptors to produce desired pharmacological effect has limited aqueous solubility. Nanoparticles by the virtue of their submicron particle size in the range of 10 to $1,000 \mathrm{~nm}$ and distinct physicochemical properties, has the ability to overcome many formulation and drug delivery issues associated with poor water solubility and lipophilicity. Preparation of nanoparticles is typically of two types either top to down approach by size reduction using conventional size reduction equipment such as media mill and high-pressure homogenizers or bottom to top formulation approaches such as precipitation, emulsion-solvent evaporation, solvent diffusion and microemulsion techniques. The critical process and formulation parameters like stirring speed, polymer and surfactant concentration can be successfully optimized to achieve desired quality attributes like particle size, zeta potential and entrapment efficiency. These parameters can further be implemented to scale-up the manufacturing process. Maintaining the stability in solution as well as in solid state without aggregation are the key factors for the successful production and scale-up of nanosuspensions. Hence the formulation of nanosuspensions as oral films stabilize them significantly by preventing aggregation while also enabling uniform resuspension upon solvation. Due to the considerable enhancement in bioavailability, adaptability for surface modification and mucoadhesion, drug targeting can significantly expand the scope of this formulation strategy. Oral film delivery of nanosuspension with receptor mediated endocytosis has the

\footnotetext{
* Corresponding author: Suhani Sinha
} 
promising ability to resolve most permeability limited absorption and hepatic first-pass metabolism related issues adversely affecting bioavailability.

\section{Current Trends in Formulation development and Research of Nanopharmaceutical Dosage Forms}

Nanopharmaceutical formulations can be classified into six types depending upon their approved clinical use like: liposome nanoparticles, polymeric nanoparticles, micelle nanoparticles, nanocrystal nanoparticles, inorganic nanoparticles and protein nanoparticles. Details of the products developed and marketed in these categories have been tabulated in Table 1.

Table FDA Approved Nanodrugs available for clinical use

\begin{tabular}{|c|c|c|c|c|}
\hline Generic name & Formulation & $\begin{array}{l}\text { Trade } \\
\text { Name }\end{array}$ & Manufacturer & $\begin{array}{l}\begin{array}{l}\text { Therapeutic Indications } \\
\text { benefits }\end{array} \\
\end{array}$ \\
\hline Doxorubicin $\mathrm{HCl}$ & $\begin{array}{l}\text { Liposome } \\
\text { nanoparticles } \\
\text { (injection) }\end{array}$ & Doxil & $\begin{array}{l}\text { Janssen } \\
\text { Pharmaceutica } \\
\text { ls }\end{array}$ & $\begin{array}{l}\text { Karposi's sarcoma, ovarian cancer, } \\
\text { multiple myeloma (Increased delivery } \\
\text { to disease site, decreased systemic } \\
\text { toxicity of free drug) }\end{array}$ \\
\hline Amphotericin B & $\begin{array}{l}\text { Liposomal } \\
\text { nanoparticles }\end{array}$ & AmBIsome & Gilead Sciences & $\begin{array}{l}\text { Fungal/protozoal infections } \\
\text { (decreased nephrotoxicity) }\end{array}$ \\
\hline Amphotericin B & $\begin{array}{l}\text { Liposomal } \\
\text { nanoparticles }\end{array}$ & Abelcet & Sigma-Tau & $\begin{array}{l}\text { Serious fungal infections (decreased } \\
\text { toxicity) }\end{array}$ \\
\hline Poractant alfa & $\begin{array}{l}\text { Liposome } \\
\text { nanoparticles }\end{array}$ & Curosurf & Chiesi USA & $\begin{array}{l}\text { Respiratory distress syndrome } \\
\text { (Increased delivery with smaller } \\
\text { volume, decreased toxicity) }\end{array}$ \\
\hline $\begin{array}{l}\text { Triamcinolone acetonide } \\
\text { ER injectable suspension }\end{array}$ & $\begin{array}{l}\text { Polymeric } \\
\text { nanoparticles }\end{array}$ & Zilretta & $\begin{array}{l}\text { Flexion } \\
\text { Therapeutics }\end{array}$ & $\begin{array}{l}\text { Osteoarthritis knee pain (extended } \\
\text { release) }\end{array}$ \\
\hline $\begin{array}{l}\text { Antihemophilic factor } \\
\text { (recombinant), pegylated }\end{array}$ & $\begin{array}{l}\text { Polymeric } \\
\text { nanoparticles }\end{array}$ & Adynovate & Shire & $\begin{array}{l}\text { Hemophilia (Greater protein stability, } \\
\text { longer half-life) }\end{array}$ \\
\hline Fenofibrate & $\begin{array}{l}\text { Nanoparticle } \\
\text { micelle }\end{array}$ & Tricor & AbbVie & $\begin{array}{lr}\text { Hyperlipidemia } & \text { (Greater } \\
\text { bioavailability } & \text { simplifies } \\
\text { administration) } & \\
\end{array}$ \\
\hline Paclitaxel & $\begin{array}{l}\text { Albumin } \\
\text { bound } \\
\text { nanoparticles } \\
\text { (iv injection) }\end{array}$ & Abraxane & $\begin{array}{l}\text { American } \\
\text { Biosciences } \\
\text { (Blauvelt, New } \\
\text { York) } \\
\end{array}$ & Metastatic breast cancer \\
\hline Sirolimus & $\begin{array}{l}\text { Nanocrystal } \\
\text { particles } \\
\text { (oral) }\end{array}$ & Rapamune & $\begin{array}{l}\text { Wyeth } \\
\text { Pharmaceutica } \\
\text { ls }\end{array}$ & $\begin{array}{llr}\begin{array}{l}\text { Immunosuppressant in } \\
\text { transplant pidney } \\
\text { bioavailability) }\end{array} & \\
\end{array}$ \\
\hline Aprepitant MK869 & $\begin{array}{l}\text { Nanocrystal } \\
\text { particles } \\
\text { (oral) }\end{array}$ & Emend & $\begin{array}{l}\text { Merck/Elan } \\
\text { (Whitehouse } \\
\text { Station, New } \\
\text { Jersey) }\end{array}$ & $\begin{array}{l}\text { For chemotherapy patients for } \\
\text { delayed nausea and vomiting (greater } \\
\text { absorption and bioavailability) }\end{array}$ \\
\hline Iron dextran & $\begin{array}{l}\text { Inorganic } \\
\text { nanoparticles } \\
\text { (injection) }\end{array}$ & Dexferrum & $\begin{array}{l}\text { (American } \\
\text { Regent) }\end{array}$ & $\begin{array}{l}\text { Iron deficiency in CKD (increased } \\
\text { dose) }\end{array}$ \\
\hline $\begin{array}{l}\text { Sodium ferric gluconate } \\
\text { complex in sucrose }\end{array}$ & $\begin{array}{l}\text { Inorganic } \\
\text { nanoparticles } \\
\text { (injection) }\end{array}$ & Ferrlecit & Sanofi-Aventis & $\begin{array}{l}\text { Iron deficiency in CKD (increased } \\
\text { dose) }\end{array}$ \\
\hline $\begin{array}{l}\text { Albumin-bound } \\
\text { paclitaxel }\end{array}$ & $\begin{array}{l}\text { Protein } \\
\text { nanoparticles }\end{array}$ & Abraxane & Celgene & $\begin{array}{l}\text { Breast cancer, NSCLC, pancreatic } \\
\text { cancer (greater solubility, increased } \\
\text { delivery to tumour) }\end{array}$ \\
\hline Denileukin diftitox & $\begin{array}{l}\text { Protein } \\
\text { nanoparticles }\end{array}$ & Ontak & Eisai & $\begin{array}{l}\text { Cutaneous T-cell lymphoma (Targeted } \\
\text { T-cell specificity, lysosomal escape) }\end{array}$ \\
\hline
\end{tabular}


The other description used in research are nanotubes, nanofibers and magnetic nano pharmaceuticals. These nanopharmaceutical dosage forms are able to accommodate the active therapeutic moiety and bio-molecules such as enzymes, vaccines, recombinant proteins, peptides, and nucleic acid for their delivery. These dosage systems have ability to improve specific targeting either passively or actively, to a particular tissue or organ of the body where they are needed. [1]

A number of potential drug candidates identified in the last few decades have excellent target receptor binding, but due to their large molecular weight and high $\log P$ values, these candidates have low aqueous solubility and thus restricts further development as a successful dosage form. It is a well-established fact that large surface area offered by particle size reduction can significantly enhance dissolution rate and bioavailability according to classical Noyes-Whitney equation. Pharmaceutical nanosuspensions of drugs are nanosized, heterogeneous aqueous dispersions of insoluble drug particles stabilized by surfactants. Accommodating large drug amount with minimum dose volume has additional benefits in parenteral and ophthalmic drug delivery system owing to the minimization of excessive use of harmful nonaqueous solvents and extreme $\mathrm{pH}$. Other advantages include increased stability, sustained release of drug, increased efficacy through tissue targeting, minimum first pass metabolism and deep lung deposition. The method of preparation, dosage forms, components and applications of nanosuspensions in drug delivery systems are schematically represented in Fig. 1. Despite the intricacies associated manufacturing, selecting appropriate unit operation, equipment and process optimization can counteract these complexities to larger extent.

Chitosan is the second most abundant next to cellulose, naturally occurring amino polysaccharide, derived as a deacetylated form of chitin. Being nontoxic, biocompatible, antibacterial and biodegradable they have significant use in research towards biomedical and pharmaceutical applications, such as drug delivery, tissue engineering, wound-healing dressing etc [2]. They have various applications for the treatment of cancer, gastrointestinal diseases, pulmonary diseases, drug delivery to the brain and ocular infections through non-parenteral route of drug delivery [3] The primary amine group in chitosan are responsible for its cationic nature, muco-adhesion, in situ gelation, antimicrobial, permeation enhancement etc. This review article discusses the various forms of polymers as oral film matrix and drug delivery device incorporating drug as chitosan nanoparticles and reports the vast literature available in drug delivery applications.

Yin et al fabricated a novel smart drug delivery system consisting of protein and chitosan nanoparticles in bilaminated films composed of the mucoadhesive chitosan-ethylenediaminetetraacetic acid hydrogel layer and the hydrophobic ethyl cellulose layer. Nanoparticles in film was characterized by electron microscopy and fluorescence microscopy, and the results showed the solid, spherical nanoparticles dispersed evenly in the porous structures of films. The properties of nanoparticles and films were investigated. The mucoadhesive force and the toxicity of NP-Film were also evaluated. It was observed that the nanoparticles could reversibly open the tight junction of the intestine and inhibit trypsin activity. The drug delivery system possessed high mucoadhesive force and low intestinal toxicity. Therefore, the NPFilm would be a promising delivery carrier for protein drugs via oral administration [4].

Pintadoa et al prepared alginate beads of bioactive molecules in guar-gum films as a single and more effective delivery system to study oral delivery of bioactive molecules. He performed a response surface method to obtain optimal formulations of alginate beads to be incorporated into guar gum oral films as combined buccal and oral delivery systems for caffeine delivery. The formulation was further characterized regarding physicochemical properties, drug release, cell viability and buccal permeability. Beads had an average size of $3.37 \pm 6.36 \mu \mathrm{m}$. Film thickness was set to $62 \mu \mathrm{m}$. Scanning electron microscopy revealed that beads were evenly distributed onto the film matrix. Evaluation of Fouriertransform infrared spectra did not indicate the formation of new covalent bonds between the matrix of guar-gum films, alginate beads and caffeine. MTT assay, performed on human buccal carcinoma TR146 cell line, revealed that alginate beads incorporated in guar-gum did not compromise cell viability after $12 \mathrm{~h}$ of exposure. As demonstrated by in vitro permeability assay using TR146 human buccal carcinoma cell lines, combination of guar-gum films and alginate beads exhibited a slower drug release, lower apparent permeability $(1.15 \mathrm{E}-05 \pm 3.50 \mathrm{E}-06)$ than for caffeine solution (2.68E$05 \pm 7.30 \mathrm{E}-06)$, guar-gum film (3.12E-05 $\pm 4.70 \mathrm{E}-06)$ or alginate beads $(2.01 \mathrm{E}-05 \pm 3.90 \mathrm{E}-06)$. Thus it was concluded that the conjugation of alginate beads within an orodispersible film matrix is an effective controlled release buccal delivery system with enhanced mucoadhesion and potential to improve in vivo bioavailability of carried drugs [5]. 


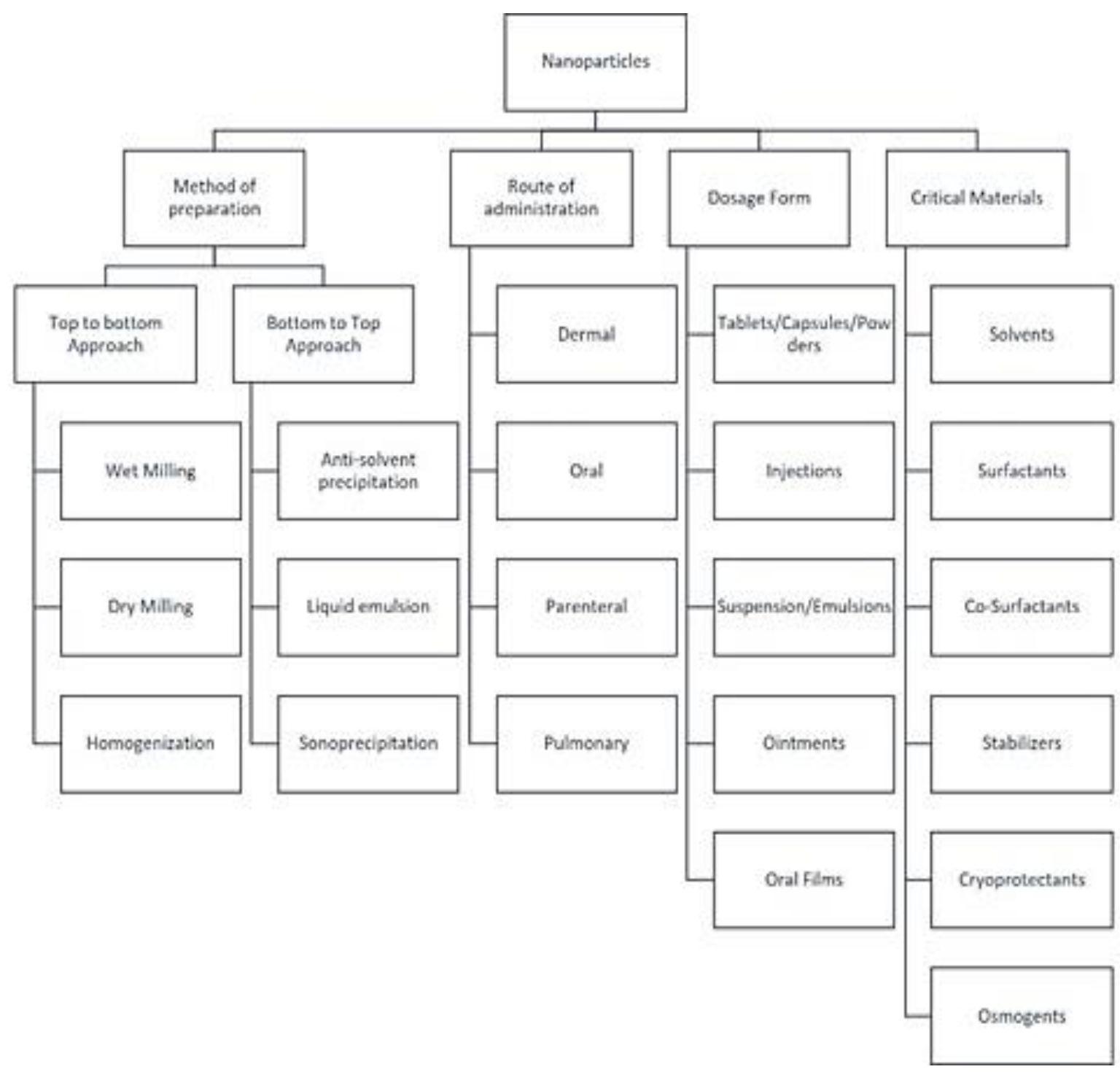

Figure 1 Method of preparation, Dosage forms, Components and Nanoparticles Applications in Drug Delivery Systems

Although several niche small molecule products have been approved as simple sprays, tablets and oral films, the concept of existing technologies cannot be applied to big molecules like biologics. Buccal delivery of insulin has reached clinical trials with two approaches; one being oromucosal spray of the peptide with permeation enhancers, and other being insulin embedded gold nanoparticles in a dissolvable film. However, neither of these approaches have led to FDA approvals likely due to submaximal peptide loading in the dosage form, and to wide intra-subject variability in pharmacokinetics and pharmacodynamics. However, it is likely that 3D printed films with lower molecular weight stable biotech payloads including lipophilic glucagon-like 1 (GLP-1) agonists and macrocytes with long half-lives will generate greater efficacy than was achieved to date for insulin [6].

In yet another study, Bao-de et al prepared a novel orodispersible film (ODF) containing Herpetrione, (a novel and potent antiviral agent with poor water solubility extracted from Herpetospermum caudigerum) nanoparticles with the goal of transforming drug nanosuspensions into a solid dosage form with enhanced oral bioavailability of drugs with poor water solubility. Nanosuspensions were prepared by high pressure homogenization and then transformed into ODF containing drug nanoparticles by mixing with hydroxypropyl methylcellulose solution containing microcrystalline cellulose, low substituted hydroxypropyl cellulose and PEG-400 followed by film casting and drying. They observed that the uniformity of dosage units of the preparation was within acceptable limits. The disintegration time observed was within $30 \mathrm{~s}$ with reconstituted nanosuspensions particle size of $280 \pm 11 \mathrm{~nm}$, which was similar to that of prepared drug nanosuspensions, indicating a good redispersibility of the fast dissolving film. X-ray diffraction showed that HPE in the orodispersible film was in the amorphous state. Increased in vitro dissolution was observed for the drug. In vivo pharmacokinetic study in rats, compared to HPE coarse suspensions, the ODF containing HPE nanoparticles exhibited significant increase in $\mathrm{AUC}_{0-24 \mathrm{~h}}, C_{\max }$ and decrease in $T_{\max }$, MRT. The result concluded that the orodispersible films 
containing drug nanoparticles have a potential opportunity to stabilize the nanoparticles and prevent agglomeration transforming drug nanosuspensions into a solid dosage form by simple process integration as well as improve bioavailability of poorly water-soluble drugs [7] [8]

Mucoadhesive films containing curcumin-loaded nanoparticles were developed, aiming to prolong the residence time of the dosage form in the oral cavity and to increase drug absorption through the buccal mucosa. Films were prepared by the casting method after incorporation of curcumin-loaded chitosan-coated polycaprolactone nanoparticles into plasticized chitosan solutions. Different molar masses of mucoadhesive polysaccharide chitosan and plasticizer glycerol were optimized during formulation development. Films obtained using medium and high molar mass chitosan were found to be homogeneous and flexible. Curcumin-loaded nanoparticles were uniformly distributed on the film surface, as evidenced by atomic force microscopy and high-resolution field-emission gun scanning electron microscopy images. Films displayed swelling of around $80 \%$ in simulated saliva and exhibited a prolonged-controlled delivery of curcumin in vitro. These results indicate that the mucoadhesive films containing nanoparticles offer a promising approach for buccal delivery of curcumin, which may be particularly useful in the treatment of periodontal diseases that require a sustained drug delivery [9].

Lercanidipine, a vasoselective dihydropyridine calcium antagonist, used for the treatment of hypertension and angina pectoris suffers from food effect, poor solubility, low permeability and considerable first pass metabolism, resulting in highly variable and low bioavailability of $10 \%$. Nanoparticles of lercanidipine were incorporated in fast dissolving oral films (FDO) via preparation of nanosuspension by evaporative antisolvent precipitation method. Prepared nanosuspensions were incorporated in films without lyophilizing or spray drying. PEG 400 and TPGS 1000 were selected as stabilizers further for incorporation in film. Physicochemical and mechanical properties of the optimized films were observed to be within acceptance criteria. SEM images as well as FTIR chemical images of oral films showed uniform distribution of nanoparticles in polymeric matrix. The DSC and XRD results proved the poorly crystalline nature of lercanidipine. It was observed that since thermal processing of film induces crystallinity in hypromellose, amorphous drug nanoparticles were embedded in semicrystalline polymeric matrix. Superior dissolution and permeability properties of nanoparticles were confirmed during in vitro dissolution studies and about 4.5-folds higher ex vivo drug permeation was observed from formulation through porcine buccal mucosa. This may give the clue for enhancement of bioavailability in vivo via improving orotransmucosal absorption [10].

Fenofibrate nanocrystal was prepared by wet media milling and formulated into oral strip-films. Mechanical properties, redispersion study, and solid-state characterization results revealed that reduction of drug crystal size at nanoscale and incorporation into oral soluble films does not affect the solid-state properties of the drug. In vitro dissolution kinetics showed enhanced dissolution rate which could be easily manipulated by changing the thickness of the film. In vivo pharmacokinetics in rabbits showed a significant difference in the pharmacokinetics parameter (1.4-fold increase bioavailability) of fenofibrate nanocrystal loaded oral soluble films as compared to the marketed formulation Tricor. This approach of drug nanocrystallization and incorporation into soluble polymeric films have significant applications in cost-effective tools for bioavailability enhancement of fenofibrate [11].

Midazolam nanosuspensions were prepared using the ultrasonic method and then incorporated in the hydroxypropyl methyl cellulose (HPMC)/pullulan polymeric matrix with other excipients like glycerol and cellulose nanofiber as a softener and a compatibilizer, respectively. The prepared films were evaluated for mechanical properties, morphology study, disintegration time, and dissolution time. SEM images of fast dissolving films showed uniform distribution of spherical nanoparticles in the polymeric matrix. A film with 36\% HPMC, 64\% pullulan, and 21\% glycerine was selected as the optimum formulation by the Design Expert 7 software. The optimum film was stable for three months. The pharmacokinetic parameters of midazolam oral film in comparison to coarse midazolam suspension exhibited significant increase in AUC, Cmax, and a significant decrease in Tmax. The overall results showed enhanced in vivo orotransmucosal absorption of poorly water-soluble drugs via the insertion of drug nanosuspension in buccal films [12]. These fast dissolving films containing midazolam deliver drugs rapidly to the blood circulatory system and have potential advantages in control of severe neuropathic attacks in children.

In another study Vaishali et al stabilized optimized nanosuspensions of glimepiride by solidification in a novel oral thin film formulation. Nanosuspensions were characterized for particle size, zeta potential as well as in vitro dissolution profile. Prepared film formulations were evaluated for physicochemical parameters like folding endurance, disintegration time, tensile strength, in vitro drug release, in vivo bioavailability and stability. It was observed that the mean particle size of the nanoparticles in oral films was found to be $57.2 \mathrm{~nm}$. In vivo bioavailability studies revealed high plasma drug concentrations (Cmax) for nanosuspension of glimepiride loaded oral films i.e. $4900 \mathrm{ng} / \mathrm{ml}$ as compared to marketed oral formulation (Cmax $-2900 \mathrm{ng} / \mathrm{ml}$ ). Results of the stability studies indicated that nanosize of 
the particles was retained even after 3 months of the study. They concluded that oral thin film formulation has a potential for stabilization of nanosuspensions [13].

Lutein is widely used as diet supplement for prevention of age-related macular degeneration. However, its application has been hampered due to its poor solubility and low oral bioavailability. A study aimed to develop and evaluate the formulation of oral fast-dissolving film containing lutein nanocrystals for enhanced bioavailability was carried out by Chen Liu et al. Lutein nanocrystals were prepared by anti-solvent precipitation method and then encapsulated into the films by solvent casting method. The formulation of oral films was optimized by Box-Behnken Design thereby using HPMC 2.05\% (w/v), PEG 400 1.03\% (w/v) and Cremophor EL 0.43\% (w/v). The obtained films exhibited uniform thickness of $35.64 \pm 1.64 \mu \mathrm{m}$ and drug content of $0.230 \pm 0.003 \mathrm{mg} / \mathrm{cm} 2$ and disintegrated rapidly in $29 \pm 8 \mathrm{~s}$. The nanocrystal-loaded films with reconstituted particle size of $377.9 \mathrm{~nm}$ showed better folding endurance and faster release rate in vitro than the conventional oral films with raw lutein. The microscope images, thermograms, and diffractograms indicated that lutein nanocrystals were highly dispersed into the films. After administrated to rats, $t$ max was decreased from $3 \mathrm{~h}$ for oral solution formulation to less than $0.8 \mathrm{~h}$ for oral film formulations, and Cmax increased from $150 \mathrm{ng} / \mathrm{mL}$ for solution to $350 \mathrm{ng} / \mathrm{mL}$ for conventional oral films or $830 \mathrm{ng} / \mathrm{mL}$ for nanocrystal in oral films. The AUC $0-24 \mathrm{~h}$ of conventional or nanocrystal oral film was 1.37 or 2.08 -fold higher than that of the oral solution, respectively. These results suggested that drug nanocrystal-loaded oral film can be applied as a promising approach for enhanced bioavailability of poor soluble drugs like lutein [14].

Another study aimed at developing fast dissolving oral films containing nanoparticles of buspirone hydrochloride was prepared. The concept of this formulation provides the drug delivery system with enhanced bioavailability and sustained release action. These formulations deliver the drug through oral mucosa and bypass the first pass metabolism thereby increase bioavailability. Nanoparticles were prepared by nanoprecipitation method and films were prepared by a solvent casting method. Both the formulations were optimized employing central composite design. Nanoparticles were evaluated for particle size, zeta potential \& entrapment efficiency, which were found to be $189.23 \pm 0.95 \mathrm{~nm},-21.56$ $\pm 0.56 \mathrm{mV}$ and $68.28 \pm 3.69 \%$, respectively. Morphological characters were identified by scanning electron microscopy, transmission electron microscopy and atomic force microscopy. Solid state characteristic of the drug in the formulation was evaluated by X-ray diffraction. Films were evaluated for their disintegration time, weight variation, thickness, tensile strength, and surface $\mathrm{pH}$. The results concluded that the nanoparticles of buspirone incorporated into films had good physicomechanical properties and optimum stability. They exhibited an initial burst release followed by sustained drug release as visualized in chronotherapy [15].

Quing Song et al prepared and evaluated fast dissolving sublingual films containing meloxicam nanocrystals. Nanocrystals were utilized to allow for improved dissolution of the drug, whereas the films were used to shorten the onset of action via the sublingual route. Meloxicam nanocrystals were prepared by nanoprecipitation based on acidbase neutralization method and then encapsulated into the films by solvent casting method. The obtained fast dissolving films exhibited uniform thickness of $82 \pm 8 \mu \mathrm{m}$ and drug content of $7.38 \pm 0.011 \mathrm{mg} /$ film $\left(4 \mathrm{~cm}^{2}\right)$ and disintegrated rapidly in $23.08 \pm 1.76 \mathrm{~s}$ with reconstituted particle size of $196.4 \pm 6.3 \mathrm{~nm}$ showed faster dissolution rate in vitro than the meloxicam coarse suspension-based films. The SEM images and XRD indicated that meloxicam nanocrystals were highly dispersed into the films. After administrated to rats, Tmax of meloxicam was significantly shortened following sublingual administration of meloxicam nanocrystals in oral films. The absorption resulted in a 3.00-fold increase in bioavailability as compared to meloxicam coarse suspension films and 4.34 times with reference to the meloxicam coarse suspension. These results indicated that the meloxicam nanoparticles in film could be a promising approach to enhance the dissolution and shorten the onset time for anti-inflammatory and analgesic drug action [16].

Attempting to overcome limitations as low bioavailability, an antihypertensive peptide derived from whey protein, with KGYGGVSLPEW sequence, was incorporated into polymer as nanoparticles. An experimental design was followed in order to optimize drug-loading, association efficiency, mean particle size, zeta-potential and polydispersity index of a formulation of poly (lactic-co-glycolic acid) (PLGA) nanoparticles as carriers for bioactive peptides. In sequence, peptide-loaded PLGA nanoparticles were incorporated in a guar-gum film matrix, resulting in a combined delivery system aiming to promote slow release and permeation across buccal epithelium. Neither PLGA nanoparticles, guargum films nor the conjugation of PLGA nanoparticles and guar-gum films (GfNp) significantly compromised in vitro TR146 human buccal carcinoma cell line viability after $12 \mathrm{~h}$ contact, as assessed by 3-(4,5-Dimethylthiazol-2-yl)-2,5Diphenyltetrazolium Bromide reduction assay (MTT). In vitro release assay for nanoparticles in film formulations revealed that the combination of orodispersible film and nanoparticles provided a sustained release of peptide when compared with control. The formulation offered more effective, synergistic, in vitro permeation of TR146 cell multilayer in comparison with guar-gum films or PLGA nanoparticles alone. The combination of PLGA nanoparticles with guargum films represent a suitable alternative to conventional delivery systems, leading to an increased buccal permeability of carried antihypertensive peptide [17]. 


\section{Toxicity Concerns with Nanopharmaceuticals}

Along with endless opportunities in the field of pharmaceutical formulation, drug delivery, diagnostic and treatment of various diseases, the safety and toxicity parameters of nano based dosage formulations is a challenge for drug development and commercialization [18]. Various semiconductive metals like colloidal gold and iron oxide crystals formulated in nano form have also demonstrated toxicity. Being submicron sized particles, they easily penetrate the skin, lungs and blood brain barrier causing toxicity. Therefore, more extensive preclinical study should be carried out to fully investigate any toxicity associated with these formulations which is a matter of concern to pharmaceutical scientists.

\section{Future Scope of Nanopharmaceuticals}

In future major share of pharmaceutical and biopharmaceutical research and commercialization shall be based using this technology of loading nanoparticles in oral films. Nanoscale formulation design and multilayer or single layer 3D printed oral film pharmaceutical dosage prepared using simple process integration without using expensive operations like lyophilization or freeze drying will be preferred choice in research to sustain the growth and market share. According to a new report published by vision gain forecasts the world market for nano pharmaceuticals has a potential to approach $\$ 130$ billion. This is due to the increasing demand of nanopharmaceutical formulations for enhanced safety and efficacy in the pharmaceutical and biopharmaceutical companies.

\section{Conclusion}

A summary of research works done on nanoformulations in oral films reveal that this technology enables conversion of poorly soluble and less bioavailable drugs into promising novel drug delivery systems with enhanced stability circumventing the first pass metabolism making it more bioavailable, while also increasing the product lifecycle. Formulation optimization with different biocompatible polymers and different molar concentrations demands a quality by design approach for a better understanding of the influence of different independent factors like polymer concentration and unit operations on the critical quality attributes of nanoparticles like particle size, zeta potential, agglomeration, drug loading and mechanisms of biological interactions. A simple process integration of nanoparticles in suspension with solvent casting technology to prepare oral films is economical with less environmental burden. In future, nano pharmaceuticals or nanoparticles in film will greatly influence healthcare as they have ability to shorten the time to market for active biologics, extend the market exclusivity of proprietary chemical drugs and create additional revenue system. Future scope is to develop nanoparticles in films for proteins and peptides, diagnostic kits, vaccines, site specific targeting of existing chemical entities and chronotherapy. One formidable challenge includes potential toxicity concern of nanoparticles as they have a tendency to accumulate in specific organs like lungs and brain. This adversity could be modified as opportunity to selectively target the drug molecules to these organs. Another challenge is the regulatory guidelines for the product registration including the manufacture and conduct of pre-clinical studies, which are not adequately addressed. A few nano-formulations in the market approved by the FDA have paved the way for further research using this tool for drug design. However, much deliberation is needed to turn the concept of nanoparticle technology into realistic application.

\section{Compliance with ethical standards}

\section{Acknowledgments}

The author is sincerely grateful to G.D.Goenka University for supporting this research work.

\section{Disclosure of conflict of interest}

The author declare that there is no conflict of interest.

\section{References}

[1] Panda BP, Patnaik S and Maharana RL. (2017). Current trends in design and development of Nanopharmaceutical dosage forms. Bioequivalence and Bioavailability International Journal, 1(1), 104.

[2] Ali A and Ahmed S. (2018). A review on chitosan and its nanocomposites in drug delivery. International Journal of Biological Macromolecules, 109, 273-286. 
[3] Mohammed MA, Syeda JTM, Wasan KM and Wasan EK. (2017). An Overview of Chitosan Nanoparticles and Its Application in Non-Parenteral Drug Delivery. Pharmaceutics, 9(4), 53.

[4] Cui F, He C, Yin L, Qian F, He M, Tang C and Yin C. (2007). Nanoparticles Incorporated in Bilaminated Films: A Smart Drug Delivery System for Oral Formulations. ACS Publications, 8(9), 2845-2850.

[5] Pedro M.C, Flávia S, Rui M, Victor M, Pizones RH, Ana M.R.P, Ana RM, Bruno S and Manuela E.P. (2018). Incorporation of beads into oral films for buccal and oral delivery of bioactive molecules. Carbohydrate polymers, 194, 411-421.

[6] Morales JO and Brayden DJ. (2017). Buccal delivery of small molecules and biologics: of mucoadhesive polymers, films, and nanoparticles. Current Opinion in Pharmacology, 36, 22-28.

[7] Shen BD, Shen CY, Yuan XD, Bai JX, Lv QY, Xu H, Dai L, Yu C, Han J and Yuan HL. (2013). Development and characterization of an orodispersible film containing drug nanoparticles. European Journal of Pharmaceutics and Biopharmaceutics, 85, 1348-1356.

[8] Jing-jing G, Peng-Fei Y, Jun-lan L, JinHana S, FuaShi-X, JinaShi-Y, Hai-Long Y. (2013). Development and in vivo/in vitro evaluation of novel herpetrione nanosuspension. International Journal of Pharmaceutics, 227-233.

[9] Mazzarino L, Borsali R and Lemos-Senna E. (2014). Mucoadhesive Films Containing Chitosan-Coated Nanoparticles: A New Strategy for Buccal Curcumin Release. Journal of Pharmaceutical Sciences, 103(11), 37643771.

[10] Chonkar AD, Rao JV, Managuli RS, Mutalik S, Dengale S, Jain P and Udupa N. (2016). Development of fast dissolving oral films containing lercanidipine $\mathrm{HCl}$ nanoparticles in semicrystalline polymeric matrix for enhanced dissolution and ex vivo permeation. European journal of Pharmaceutics and Biopharmaceutics, 103, $179-191$.

[11] Kevadiya BD, Barvaliya M, Zhang L, Anovadiya A, Brahmbhatt H, Paul P and Tripathi C. (2018). Fenofibrate Nanocrystals Embedded in Oral Strip-Films for Bioavailability Enhancement. Bioengineering, 5(1), 1.

[12] Soroushnai A1, Ganji F1, Vasheghani-Farahani E1 and Mobedi H2. (2018). Development and Evaluation of an Anti-Epileptic Oral Fast-Dissolving Film with Enhanced Dissolution and In vivo Permeation. Current Drug Delivery, 1294-1304.

[13] Kilor, V., Sapkal, N., Daud, A., Humne, S. and Gupta, T. (2017). Development of stable nanosuspension loaded oral films of glimepiride with improved bioavailability. International Journal of Applied Pharmaceutics, 9(2), 28-33.

[14] Chen L, Daoxiao C, Xinhui Z, Hong S, Yindi K, Rongyue Z, and Wenping W. (2017). Oral fast-dissolving films containing lutein nanocrystals for improved bioavailability: formulation development, in vitro and in vivo evaluation. AAPS PharmSciTech, 18(8).

[15] Bharti K, Mittal P and Mishra B. (2019). Formulation and characterization of fast dissolving oral films containing buspirone hydrochloride nanoparticles using design of experiment. Journal of Drug Delivery Science and Technology, 49, 420-432.

[16] Qing S, Chengying S, Baode S, Wangquan L, Xiao L, Bo Dai and Hailong Y. (2018). Development of a fast dissolving sublingual film containing meloxicam nanocrystals for enhanced dissolution and earlier absorption. Journal of Drug Delivery Science and Technology, 243-252.

[17] Castro PM, Baptista P, Madureira AR, Sarmento B and Pintado ME. (2018). Combination of PLGA nanoparticles with mucoadhesive guar-gum films for buccal delivery of antihypertensive peptide. International Journal of Pharmaceutics, 547(1-2), 593-601.

[18] CL Ventola. (2017). Progress in Nanomedicine: Approved and Investigational Nanodrugs. Pharmacy and Therapeutics, 742-755.

\section{How to cite this article}

Sinha S. (2020). Nanoparticles loaded in consumable polymeric oral films: A smart dosage form design for oral administration. GSC Biological and Pharmaceutical Sciences, 12(1), 07-14. 